Schmerz 2021 · 35:237-241

https://doi.org/10.1007/s00482-021-00548-3

Eingegangen: 7. Oktober 2020

Überarbeitet: 18. Februar 2021

Angenommen: 23. Februar 2021

Online publiziert: 9. April 2021

(c) Springer Medizin Verlag GmbH, ein Teil von Springer Nature 2021
Der Querschnittsbereich (QB) 13 Palliativmedizin ist als Pflichtlehr- und Prüfungsfach mit einem Stundenumfang von durchschnittlich 22 Unterrichtseinheiten $(\mathrm{UE}=45 \mathrm{~min})$ an allen medizinischen Fakultäten in Deutschland etabliert. Vorrangig wurden bisher Präsenzveranstaltungen genutzt, dies waren neben Vorlesungen und Seminaren der Unterricht am Krankenbett (UAK) und problemorientiertes Lernen (POL). Ein begleitendes E-Learning, insbesondere für die Bereiche Grundlagen, Symptomkontrolle und psychosoziale Aspekte, wurde hauptsächlich an Fakultäten mit Lehrstuhl genutzt. Prüfungen fanden zum überwiegenden Teil im MultipleChoice-Format statt $[1,2]$.

Durch die Coronavirus-disease2019(COVID-19)-Pandemie im Frühjahr 2020 sahen sich die Lehrenden während des laufenden Sommersemesters (SoSe) plötzlich mit der Herausforderung konfrontiert, die etablierten Präsenzveranstaltungen ad hoc durch elektronische Lehrformate ersetzen $\mathrm{zu}$ müssen.

\section{Zielsetzung}

Diese Erhebung hatte das Ziel, die Lehrsituation in der Palliativmedizin unter dem Einfluss der COVID-19-Pandemie abzubilden und möglichen Entwicklungsund Unterstützungsbedarf zu identifizieren.

\author{
Alexandra Scherg ${ }^{1,3} \cdot$ Benjamin Ilse $^{2} \cdot$ Frank Elsner $^{1}$ \\ ${ }^{1}$ Klinik für Palliativmedizin, Uniklinik RWTH Aachen, Aachen, Deutschland \\ ${ }^{2}$ Klinik für Neurologie, Universitätsklinikum Jena, Jena, Deutschland \\ ${ }^{3}$ Abteilung für Hämatologie/Onkologie, Evangelisches Krankenhaus Wesel, Wesel, Deutschland
}

\title{
Palliativmedizinische Lehre im Querschnittsbereich 13 unter dem Einfluss von COVID-19
}

\section{Methoden}

In einem ersten Schritt wurden in der Arbeitsgruppe (AG) Bildung der Deutschen Gesellschaft für Palliativmedizin (DGP) 15 Items entwickelt, die die aktuelle palliativmedizinische Lehrsituation in Deutschland abbildeten und Unterstützungswünsche an die DGP adressieren sollten. Über persönliche Kontakte und eine ergänzende Onlinerecherche wurden die Lehrverantwortlichen aller $41 \mathrm{Fa}$ kultäten identifiziert und zur Teilnahme an der Befragung eingeladen. Die Befragung wurde mit SurveyMonkey ${ }^{\circledR}$ (SurveyMonkey Europe UC, Dublin, Irland) erstellt und umfasste 7 Items, die sich auf das SoSe 2020 bezogen, 4 Items, die das Wintersemester 2020/2021 betrafen, sowie 4 allgemeine Items. Acht der zuvor beschriebenen 15 Items verlangten eine Freitextantwort, 4 Items waren in Form einer numerischen Rating-Skala (NRS) $\mathrm{zu}$ beantworten, 3 in Form einer LikertSkala.

In einem Expertenworkshop mit 14 Lehrenden verschiedener Fakultäten wurden im Anschluss zunächst eigene Erfahrungen und Einschätzungen diskutiert und in einem nächsten Schritt auf die Ergebnisse der Onlinebefragung angewendet. Die Diskussionsergebnisse wurden mit in die Auswertung einbezogen, wobei nicht auszuschließen ist, dass ein Teil der Experten auch den Fragebogen beantwortet hat.

\section{Ergebnisse}

Von 41 kontaktierten Lehrverantwortlichen bearbeiteten 16 die Umfrage, zum Teil unter Auslassen einzelner Items. Das entspricht einer Rücklaufquote von $39 \%$. In einer Beantwortung wurde die $\mathrm{Zu}$ stimmung zur Publikation der genannten Lehr- und Prüfungsformate sowie der genutzten Software verweigert, sodass diese Antworten nicht ausgewertet wurden.

Die digitale Vermittlung der relevanten Lehrinhalte wurde mehrheitlich als gelungen eingeschätzt $(n=15 ;$ NRS 0 [trifft voll zu] bis 100 [trifft gar nicht zu], Mittelwert [MW] 19, Range 0-50). Die Lehrgestaltung im SoSe 2020 wurde durch die COVID-19-Pandemie überwiegend als Herausforderung erlebt ( $n=14$; NRS 0 [trifft voll zu] bis 100 [trifft gar nicht zu], MW 26, Range 0-85). Die Umsetzung digitaler Prüfungen hingegen wurde weniger positiv erlebt ( $n=13$; NRS 0 [trifft voll zu] bis 100 [trifft gar nicht zu], MW 39, Range 0-100). Je niedriger der Wert auf der $\mathrm{Y}$-Achse, desto größer ist das $\mathrm{Maß}$ an positiver Zustimmung (• Abb. 1).

Zur Vermittlung der Struktur einer Palliativstation wurden bereits etablierte Rundgänge über Stationen als Videomitschnitt für die Studierenden angeboten. Als neu eingeführte, häufig genutzte Lehrformate wurden Onlinevorlesungen mit vertonten (bereits existierenden) PowerPoint-Folien genannt. Um diese aufzuwerten, erfolgte die Vertonung teils durch mehrere Dozenten. Vorlesungen fanden weiterhin via Zoom $^{\circledR}$ (Zoom Video Communications, Inc., San Jose, 


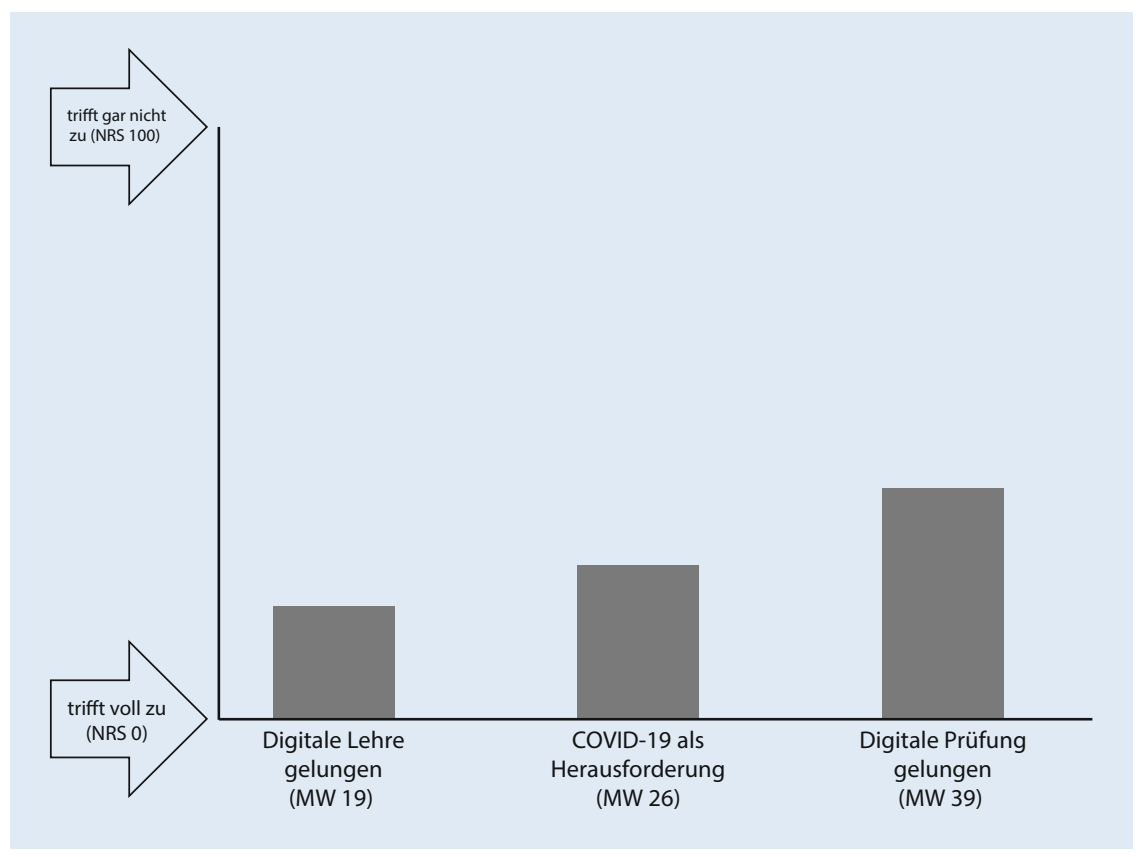

Abb. $1 \Delta$ Bewertung der Lehrgestaltung im Sommersemester 2020. MW Mittelwert, NRS numerische Rating-Skala

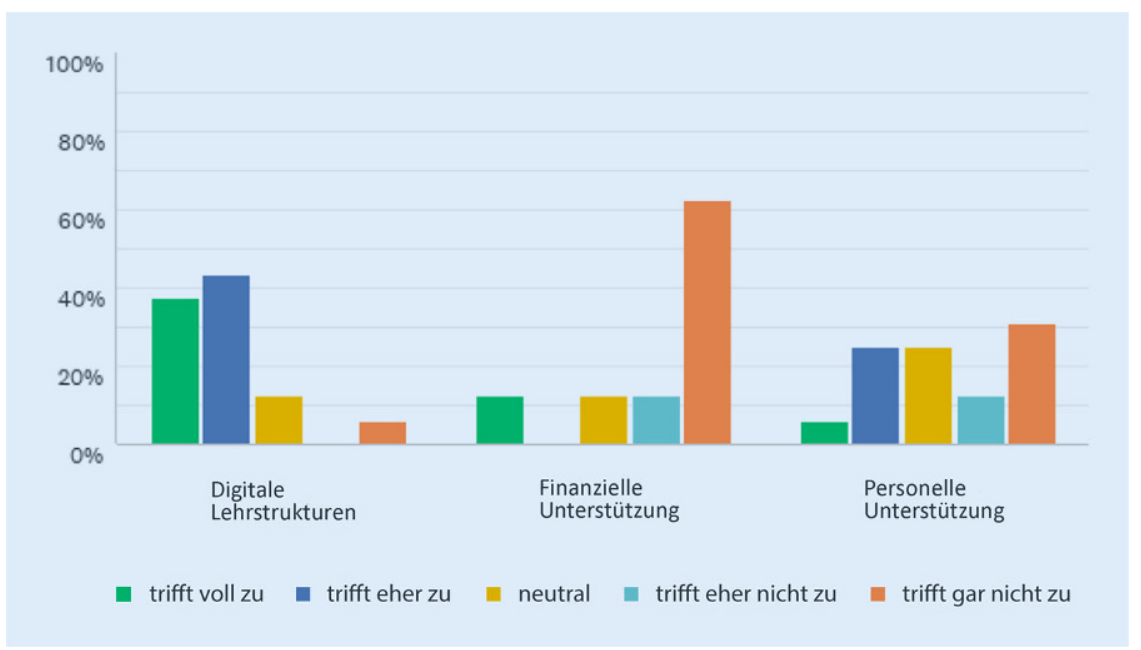

Abb. 2 \ Unterstützung seitens der Fakultäten bei der Lehrgestaltung im Sommersemester 2020

CA, USA) sowie über andere E-LearningTools statt. Auch videobasierte Seminare via Zoom $^{\circledast}$ wurden durchgeführt. Es wurden für das selbstständige Lernen Lehrtexte und Reflexionsfragen zur Verfügung gestellt. Um den Realitätsbezug herzustellen wurden teilweise Patient*innen via Videosequenz (Tablet) vorgestellt und direkte Gespräche zwischen Studierenden und Patient ${ }^{\star}$ innen mittels Video ermöglicht. Zum Kommunikationstraining wurden digitale $\mathrm{Ge}$ spräche mit Simulationspatient*innen angeboten.
Die Prüfungen fanden teils als Präsenzprüfung mit Abstandsregeln, teils als E-Klausur statt, auch eine fallbasierte Hausarbeit und Hospitationsberichte wurden genannt. Das überwiegende Prüfungsformat bestand weiterhin aus Multiple-Choice-Fragen.

Unterstützung seitens der Fakultäten wurde in erster Linie in Form von digitalen Lehrstrukturen benannt, weniger als finanzielle oder personelle Unterstützung ( $n=16$; $\bullet$ Abb. 2).

Das Feedback der Studierenden fiel hinsichtlich der vertonten Präsentatio- nen und Videos positiv aus. Kritisiert wurde neben strukturellen Aspekten, wie technischen Problemen, insbesondere die Prozessebene. Durch die Digitalisierung würde die Interaktion mit Patient ${ }^{*}$ innen und Dozierenden verloren gehen und in der häuslichen Umgebung würde es schwerer fallen, sich auf die Lehrinhalte zu konzentrieren.

Mit einer Ausnahme planten alle Teilnehmenden für das Wintersemester 2020/2021 ebenfalls eine vollständige oder teilweise digitale Lehrgestaltung. Die bereits angewendeten Lehrformate sollen überwiegend beibehalten werden, da die Umstellung mit hohem Arbeitsaufwand verbunden war. An einigen Standorten soll Kleingruppenunterricht als Präsenzlehre ermöglicht werden, um hier ein höheres Maß an Interaktion und Patientenkontakt zu ermöglichen. Die Prüfungen werden überwiegend weiterhin als Multiple-Choice-Klausur, elektronisch oder als Präsenzveranstaltung geplant, auch fallbasierte Hausarbeiten und „objective structured clinical examinations" (OSCE) sind vorgesehen.

Als Software wurden auch mit Blick auf das kommende Semester überwiegend Zoom ${ }^{\circledR}$ und die Nutzung vertonter PowerPoint-Vorträge angeführt.

An die DGP wurde der Wunsch adressiert, Lehr- und Prüfungsmaterial sowohl zentral zu erstellen als auch zu sammeln und den Fakultäten zur Verfügung zu stellen ( $n=16$; $\bullet$ Abb. 3 ).

Als Herausforderung bei der Lehrgestaltung unter dem Einfluss von COVID19 beschrieben die Lehrenden analog zum Feedback der Studierenden die fehlenden Interaktionsmöglichkeiten, insbesondere bei der Vermittlung psychomotorischer und affektiver sowie multiprofessioneller Lehrinhalte. Auch technische Hürden, fehlende Unterstützung und die Kurzfristigkeit der neuen und stetig wechselnden Anforderungen wurden kritisiert. Aber auch Chancen wurden benannt, neben der Digitalisierung selbst vor allem die Chance zur Neugewichtung der Lehrinhalte im Studium und das höhere Maß an Eigenverantwortung aufseiten der Studierenden. 
Schmerz 2021 · 35:237-241 https://doi.org/10.1007/s00482-021-00548-3

(c) Springer Medizin Verlag GmbH, ein Teil von Springer Nature 2021

\section{A. Scherg · B. Ilse · F. Elsner}

\section{Palliativmedizinische Lehre im Querschnittsbereich 13 unter dem Einfluss von COVID-19}

\section{Zusammenfassung}

Hintergrund. Aufgrund der Coronavirusdisease-2019(COVID-19)-Pandemie wurde im Sommersemester (SoSe) 2020 an allen deutschen medizinischen Fakultäten die Präsenzlehre weitestgehend eingestellt. Betroffen war auch der Querschnittsbereich (QB) 13 Palliativmedizin mit einem Umfang von durchschnittlich 22 Unterrichtseinheiten (UE). Die Lehrenden waren aufgefordert, kurzfristig digitale Lehr- und Prüfungsangebote zu entwickeln.

Ziel. Ziel dieser Erhebung war, die Lehrsituation im QB 13 Palliativmedizin im SoSe 2020 unter dem Einfluss von COVID-19 abzubilden und daraus Vernetzungs- und Unterstützungsbedarf für die kommenden Semester abzuleiten.
Material und Methoden. Die Schlüsselpersonen der palliativmedizinischen Lehre aller 41 medizinischen Fakultäten in Deutschland wurden identifiziert und von der Deutschen Gesellschaft für Palliativmedizin (DGP) zur Teilnahme an einer Onlinebefragung mit SurveyMonkey ${ }^{\circledR}$ (SurveyMonkey Europe UC, Dublin, Irland) eingeladen. Der Fragebogen bestand aus 15 Items, die sich inhaltlich mit der Evaluation des vergangenen Semesters und dem Unterstützungsbedarf des kommenden Semesters befassten. Ergebnisse. Von 41 Lehrverantwortlichen nahmen 16 an der Befragung teil. Der überwiegende Teil beschreibt die Durchführung der Lehrgestaltung im SoSe 2020 als gelungen. Unterstützung durch die Fakultäten war primär in Form digitaler Lehrstrukturen gegeben. Auch für das Wintersemester 2020/21 ist eine digitale Lehrgestaltung geplant. Häufig genutzt werden vertonte PowerPoint-Folien sowie das Konferenztool Zoom ${ }^{\circledR}$ (Zoom Video Communications, Inc., San Jose, CA, USA).

Schlussfolgerung. Die Gestaltung der Prüfungen im SoSe 2020 wird in der Selbsteinschätzung schlechter bewertet als die Lehrgestaltung, häufig fanden Präsenzprüfungen im Multiple-Choice-Format statt. Die Lehrenden wünschen sich von der DGP eine zentrale Erstellung und Sammlung von Lehr- und Prüfungsmaterialien.

\section{Schlüsselwörter}

Umfrage - Medizinstudium · Digitale Technik . Lehrmaterialien · Lockdown

\section{Undergraduate palliative care teaching in times of COVID-19}

\section{Abstract}

Background. Due to the coronavirus disease 2019 (COVID-19) pandemic classroom teaching was largely discontinued at all German medical faculties in the summer semester (SS) 2020. This also affected undergraduate education in palliative care, which is established with an average of 22 teaching units. Teachers were asked to rapidly develop digital teaching and assessment tools.

Objective. The aim of this survey was to map the teaching situation in QB13 palliative medicine in SS 2020 under the influence of COVID-19 and from this to derive the need for networking and support for the coming semesters.
Material and methods. The key persons involved in palliative care education in all 41 medical faculties in Germany were identified and invited by the coordination office of the German Society for Palliative Medicine (DGP) to participate in an online survey using SurveyMonkey ${ }^{\circledR}$. The questionnaire consisted of 15 items dealing with the evaluation of the past semester and the need for support for the coming semester.

Results. Out of 41 contacted teachers 16 participated in the survey. Most of them described the challenge of teaching in SS 2020 as successful. Support from the faculties was provided primarily in the form of digital teaching structures. A digital teaching design is also planned for the coming semester. Power point slides with sound and the conference tool Zoom ${ }^{\circledR}$ are often used. Conclusion. The design of examinations in SS 2020 is rated worse in the self-evaluation than the teaching design. Multiple choice questions were often used in classroom examinations. Lecturers would like the DGP to create and collect teaching and assessment materials centrally.

Keywords

Survey · Education, medical · Digital technology · Teaching materials · Lockdown

\section{Diskussion}

Die erhobenen Daten ergänzen die Darstellung der palliativmedizinischen Lehrsituation in Deutschland aus der aktuellen Lehrumfrage [2] um Angaben zur rasch angepassten Lehr- und Prüfungssituation unter dem Einfluss der COVID19-Pandemie. Die Rücklaufquote war mit $39 \%$ gering, was auf den kurzen Erhebungszeitraum während der Sommerzeit zurückzuführen ist. Die Inhalte deckten sich jedoch mit den Ergebnissen der anschließenden Expertendiskussion und lassen somit eine inhaltliche Sättigung vermuten.

Die technischen Grundlagen für das Erarbeiten eines digitalen Lehrangebots scheinen grundsätzlich verfügbar zu sein, und über die Anbieter selbst oder die ITAbteilungen der Fakultäten werden Schulungen angeboten. Dennoch wäre personelle Unterstützung in Form von ITExperten wünschenswert. Weitere direkte finanzielle Unterstützung erscheint bei ausreichendem Lehrpersonal eher zweit- rangig: Obwohl seitens der Fakultäten kaum unmittelbare finanzielle Unterstützung zur Lehrgestaltung geleistet wurde (- Abb. 2), war die Bewertung der Lehre überwiegend positiv (• Abb. 1).

Im Bereich der Lehre wäre es wünschenswert, zumindest einen Teil der Veranstaltungen in Kleingruppen wieder in Form von Präsenzlehre anbieten zu können oder alternativ digitale Lösungen $\mathrm{zu}$ finden, welche mehr Interaktion und die Vermittlung von Fertigkeiten und Haltung zulassen. In der Expertengruppe 


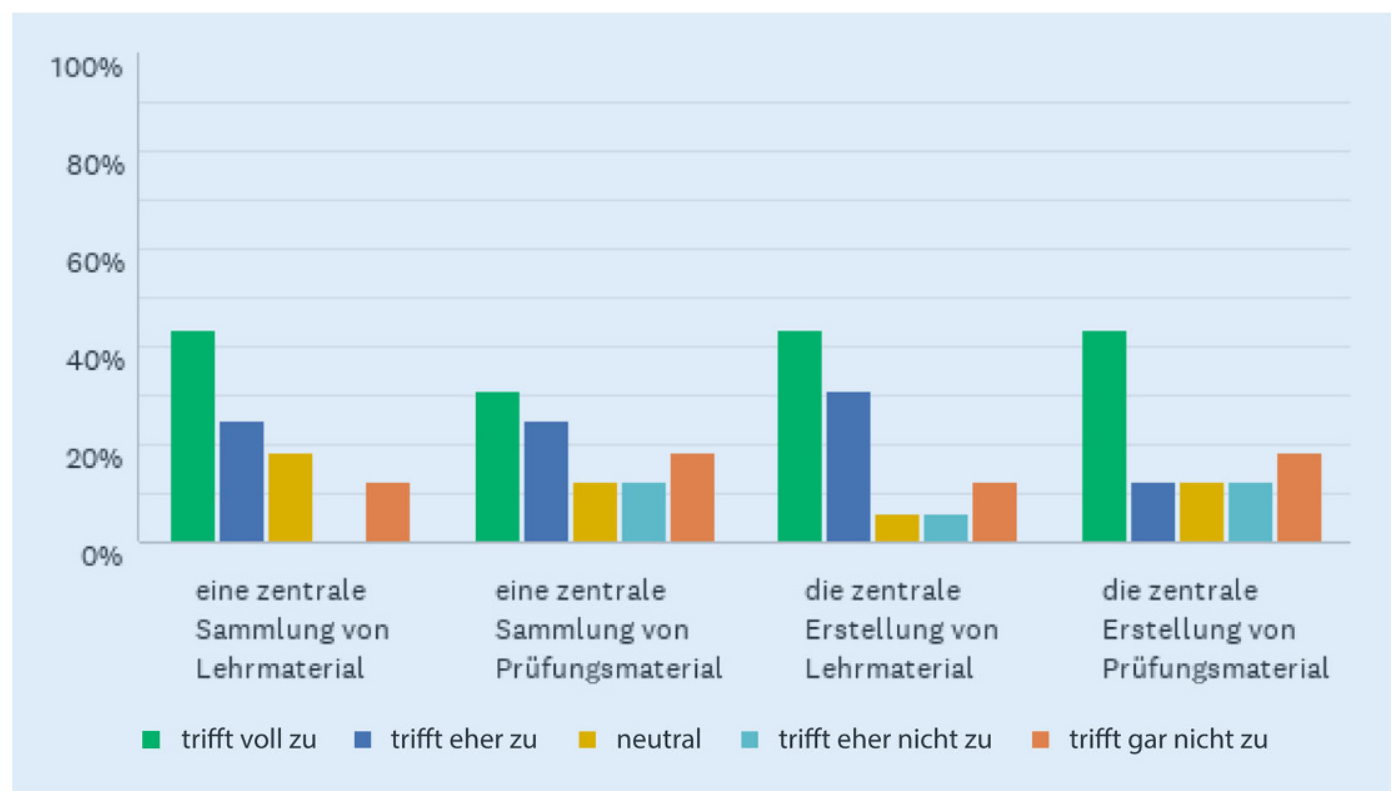

Abb. $3<$ Wünsche an die Deutsche Gesellschaft für Palliativmedizin (DGP)

wurde in diesem Zusammenhang auch diskutiert, dass die elektronischen Lehrformate eine Chance darstellen können, offener und den eigenen Bedürfnissen angepasster mit emotional herausfordernden Themen umzugehen. So bietet das digitale Lernen einen anonymeren Rahmen und reduziert so für manche Studierende die Hemmschwelle, sich auf emotionale Themen einzulassen oder offen Fragen zu stellen.

\section{》) Elektronische Lehrformate können die Chance bieten, offe- ner mit emotional herausfordern- den Themen umzugehen}

Eine größere Herausforderung scheint wie auch bisher beschrieben [1,2] die Gestaltung der Prüfungen darzustellen. In der Diskussion wurde deutlich, dass die aktuelle Situation durchaus die Chance mit sich bringt, auch die Prüfungen neu zu gestalten und elektronische Formate $\mathrm{zu}$ entwickeln, die neben der Prüfung von Faktenwissen auch psychomotorische und affektive Lernziele prüfen. Ein erster Schritt ist bereits damit getan, dass die Fakultäten sich dem rechtlich schwierigen Thema der elektronischen Prüfungen widmen (müssen). Als ein Pilotprojekt wurde in der AG Bildung der DGP bereits „Die virtuelle Palliativ- patientin“ als elektronisches Prüfungstool auf Basis einer Fallvignette entwickelt und an vier Standorten getestet [3].

\section{Ausblick}

Entsprechend dem Wunsch der Befragten soll eine bessere Vernetzung der Lehrenden mit dem Schwerpunkt des Austauschs von Erfahrungen und Lehrmaterialien ermöglicht werden. Es ist daher geplant, neben den bereits etablierten, jährlich stattfindenden QB-13-Dozierenden-Workshops (in der Regel nach Ende des SoSe) ein zusätzliches, virtuelles Treffen am Ende des Wintersemesters via Zoom $^{\circledR}$ zu veranstalten.

Darüber hinaus wurde im Mitgliederbereich der DGP die bereits vorhandene Moodle ${ }^{\circledR}$-Plattform (eLeDia eLearning im Dialog GmbH, Berlin, Deutschland) zum Austausch von Lehrmaterial aktualisiert. In Zukunft sollen dort bereits vorhandene, aber auch neu generierte Filmsequenzen, Fallvignetten etc. zur freien Nutzung im Rahmen der Lehre bereitgestellt werden.

Parallel soll das Tool „Die virtuelle Palliativpatientin“ weiterentwickelt werden und im Verlauf als technisches Gerüst dienen, das die einzelnen Fakultäten mit Inhalten (Multiple-Choice-Fragen, Filmsequenzen, Reflexionsfragen) füllen können.

\section{Korrespondenzadresse}

\section{Alexandra Scherg}

Abteilung für Hämatologie/Onkologie, Evangelisches Krankenhaus Wesel

Schermbecker Landstraße 88, 46485 Wesel, Deutschland

scherg@evkwesel.de

\section{Einhaltung ethischer Richtlinien}

Interessenkonflikt. A. Scherg, B. Ilse und F. Elsner geben an, dass kein Interessenkonflikt besteht.

Alle beschriebenen Untersuchungen am Menschen oder an menschlichem Gewebe wurden mit Zustimmung der zuständigen Ethikkommission, im Einklang mit nationalem Recht sowie gemäß der Deklaration von Helsinki von 1975 (in der aktuellen, überarbeiteten Fassung) durchgeführt. Es liegt ein positives Votum der Ethikkommission der Medizinischen Fakultät der RWTH Aachen (EK 300/20) vor.

\section{Literatur}

1. Ilse B, Alt-Epping B, Kiesewetter I, Elsner F, Hildebrandt J, Laske A, Scherg A, Schiessl C (2015) Undergraduate education in palliative medicine in Germany: a longitudinal perspective on curricular and infrastructural development. BMC Med Educ 15:151. https://doi.org/10.1186/ s12909-015-0439-6

2. OhImeier L, Scherg A, Ilse B, Elsner F (2021) Entwicklung der palliativmedizinischen Lehre in Deutschland. Schmerz. https://doi.org/10.1007/ s00482-021-00536-7

3. Scherg A, Oechsle K, Coym A, llse B, Annweiler B, Alt-Epping B, Neukirchen M, Lemos M, Stummer T, Seibel J, Elsner F (2021) Die virtuelle Palliativpatientin - Pilotierung eines elektronischen Tools zur Prüfung kognitiver, psychomotorischer und 


\section{Fachnachrichten}

affektiver palliativmedizinischer und schmerztherapeutischer Lerninhalte während der ärztlichen Ausbildung. Schmerz. Manuskriptnummer DSCHD-20-00050R1

\section{Das Dilemma mit den Meta-Analysen am Beispiel Rücken- schmerz}

\section{Stellungnahme des Wissenschaftszentrums der Deutschen Ärztegesellschaft für Akupunktur}

Im Dezember letzten Jahres wurde von $\mathrm{Mu}$ et al. eine aktualisierte Cochrane-Meta-Analyse der Studienlagen zum Nutzen der Akupunktur bei chronischen Rückenschmerzen publiziert [2]. Die Autoren analysieren 33 Studien mit 8270 Patienten. Nadelinterventionen mit Akupunktur wird im Vergleich zu keiner Intervention eine bessere Wirksamkeit in der unmittelbaren Schmerzreduktion und Funktionsverbesserung zugeschrieben. Im Vergleich zu Standardschmerzbehandlungen verbessert eine Nadelakupunktur insbesondere die körperliche Funktion und die physische Komponente der Lebensqualität. Die Autoren können zudem einen statistischen Unterschied zu Sham-Akupunkturinterventionen feststellen. Die klinische Bedeutsamkeit wird jedoch als gering beurteilt. Die Autoren geben die Sicherheit ihrer Evidenzeinschätzung mit moderat bis sehr niedrig an, dies auch in Folge eines vermeintlichen hohen Bias-Risikos der eingeschlossenen Studien.

Die online-publizierte Stellungnahme des Wissenschaftszentrum [3] widmet sich insbesondere dieser Lesart, wie sie auch in einer Newsmeldung des Deutschen Ärzteblattes [1] aufgegriffen wurde. Dabei werden von den Autoren die Fragen nach der Verblindung in Akupunkturstudien - also dem Vergleich zu Schein-Akupunktur - der Zahl der Studienabbrecher in der Nachbeobachtung nach einem Jahr, aber auch einer vermeintlich ungenügenden klinischen Relevanz, über die Maßen als Negativkriterien angelegt. Dies kontrastiert vor dem Hintergrund weiterer Cochrane-Meta-Analysen zum Thema Rückenschmerz, aber auch anderen themenverwandten Analysen, in welchen ein anderer Maßstab angelegt wurde.

Dass die Akupunktur gegenüber einer NichtBehandlung einen relevanten klinischen Wirksamkeitsvorteil erzielt, und somit chronischen Schmerzpatienten effektiv zu helfen vermag, wird von den Autoren nicht entsprechend dargestellt.

\section{Literatur}

1. Hil/Aerzteblatt.De (2021) Was Akupunktur bei chronischen Rückenschmerzen bringt. https://www.aerzteblatt.de/nachrichten/ 120467/Was-Akupunktur-bei-chronischen-Rueckenschmerzen-bringt (letzter Zugriff 19. Februar 2021)

2. Mu J, Furlan AD, Lam WY et al. (2020) Acupuncture for chronic nonspecific low back pain. The Cochrane database of systematic reviews 12:Cd013814

3. Fleckenstein J, Banzer W. (2021) Das Dilemma mit den Meta-Analysen am Beispiel Rückenschmerz - Stellungnahme des Wissenschaftszentrums der Deutschen Ärztegesellschaft für Akupunktur. Deutsche Zeitschrift für Akupunktur. (epub ahead of print) https://dx.doi.org/10.1007/s42212-02100363-y

\section{Quelle: Wissenschaftszentrums der Deutschen Ärztegesellschaft für Akupunktur}

